\title{
Potential of Sentinel-1 and Sentinel-2 data for Mapping Irrigated areas at plot scale
}

\author{
Hassan Bazzi, Nicolas Baghdadi, Dino Ienco, Mehrez Zribi, Hatem Belhouchette
}

Irrigation plays a significant role in agricultural production in order to meet the global food requirement under changing climatic conditions. To fulfil the high demand of food with an ever-increasing global population, better planning of irrigation is required. Therefore, more focus is being set on the assessment of irrigation performance for improving water management in order to achieve higher water productivity and increase the agricultural water sustainability.

In the context of mapping irrigated areas, we propose an innovative approach to map irrigated areas using Sentinel-1 (S1) SAR (Synthetic Aperture Radar) and Sentinel-2 (S2) optical time series. Our proposed approach is based on the use of S1 and/or S2 time series combined with statistical and mathematical functions such as principal component analysis (PCA) and wavelet transformation (WT). The proposed approach was tested over the Catalonia region, Spain with a dataset containing 126000 irrigated and 67000 non-irrigated plots. The novelty of our study resides in eliminating the ambiguity between irrigation and rainfall by comparing between the SAR backscattering signal of each plot and that of the corresponding grid $(10 \mathrm{~km} \times 10 \mathrm{~km})$. The potential of S2 images to classify irrigated areas by means of NDVI time series was also investigated in this study. Random forest $(\mathrm{RF})$ and convolutional neural network $(\mathrm{CNN})$ approaches were used to build up classification models using the PCA or WT parameters in three different scenarios: The first using only S1 data, the second using only S2 data, and the third using both S1 and S2 data.

The RF classifiers built using the PCA or WT on S1 time series perform well in mapping irrigated areas with an accuracy of $90.7 \%$ and $89.1 \%$ respectively. However, the CNN classification on the S1 temporal series produces a significant overall accuracy of $94.1 \%$. Finally, the combined use of the SAR and optical data enhanced the accuracy of the RF classification but did not significantly change the overall accuracy of the CNN model. 